\title{
COMPOSIÇÃO MINERAL E SEVERIDADE DE "BITTER PIT" EM MAÇÃS 'CATARINA'1
}

\author{
CASSANDRO VIDAL TALAMINI DO AMARANTE ${ }^{2}$, DANIELA VIEIRA CHAVES ${ }^{3}$, PAULO ROBERTO ERNANI
}

RESUMO - Maçãs 'Catarina', colhidas na maturação comercial em pomar no município de São Joaquim-SC, foram separadas em quatro lotes de 14 frutos, de acordo com a severidade de incidência de "bitter pit": nula (nenhuma lesão/fruto), baixa (1-2 lesões/fruto), moderada (3-5 lesões/fruto) e alta (6-18 lesões/fruto). Foram determinadas as concentrações de $\mathrm{Ca}, \mathrm{Mg}, \mathrm{K}$ e N na casca e na polpa de cada fruto. Foram verificadas relação linear (P < $0,05)$ negativa entre os valores médios de Ca na casca, e positiva entre as relações $\mathrm{Mg} / \mathrm{Ca},(\mathrm{K}+\mathrm{Mg}) / \mathrm{Ca}$ e $(\mathrm{K}+\mathrm{Mg}+\mathrm{N}) / \mathrm{Ca}$ também na casca, com o número médio de manchas/fruto nas diferentes categorias de severidade de "bitter pit". Para a polpa, o aumento na severidade de "bitter pit" foi acompanhado de redução apenas nas concentrações de Ca e Mg. A análise multivariada (análise canônica discriminante) mostrou que a relação Mg/ Ca na casca foi o atributo mineral que melhor discriminou frutos com diferentes níveis de severidade ao "bitter pit", e que, portanto, elevados valores desta relação são indicativos de frutos com alta suscetibilidade a esse distúrbio na cultivar Catarina.

Termos para indexação: Malus domestica Borkh., nutrição, distúrbio fisiológico, pós-colheita.

\section{MINERAL COMPOSITION AND BITTER PIT SEVERITY IN 'CATARINA' APPLES}

\begin{abstract}
Catarina' apples were harvested at the commercial maturity in an orchard in São Joaquim-SC and segregated in four lots of 14 fruits with different levels of bitter pit severity: null (none pit/fruit), low (1-2 pits/fruit), moderate (3-5 pits/fruit), and high (6-18 pits/fruit). Nutritional analysis (Ca, $\mathrm{Mg}, \mathrm{K}$, and $\mathrm{N}$ ) in the skin and flesh tissues were performed on individual fruits of each severity level. The average number of pits/fruit (calculated for each lot of bitter pit severity) showed a negative linear relationship $(\mathrm{P}<0.05)$ with the skin $\mathrm{Ca}$ content, and a negative linear relationship $(\mathrm{P}<0.05)$ with the ratios of $\mathrm{Mg} / \mathrm{Ca},(\mathrm{K}+\mathrm{Mg}) / \mathrm{Ca}$, and $(\mathrm{K}+\mathrm{Mg}+\mathrm{N}) / \mathrm{Ca}$ in the skin. For the flesh, the increasing of bitter pit severity was accompanied by significant reduction of $\mathrm{Ca}$ and $\mathrm{Mg}$ contents. The multivariate analysis (canonical discriminant analysis) showed that the $\mathrm{Mg} / \mathrm{Ca}$ ratio in the skin provided the best discrimination between the lots of fruit with different levels of bitter pit severity. Therefore, for 'Catarina' apples, increasing values of the $\mathrm{Mg} / \mathrm{Ca}$ ratio in the skin are indicative of fruits with increasing bitter pit susceptibility.
\end{abstract}

Index terms: Malus domestica Borkh., nutrition, physiological disorder, postharvest.

\section{INTRODUÇÃO}

A cultura da maçã tem importante papel na economia brasileira. Todavia, o elevado volume de frutos produzidos exige especial atenção no tocante à preservação da qualidade pós-colheita durante o armazenamento. Muitos esforços têm sido feitos para preservar a qualidade pós-colheita em maçãs, através de medidas visando a retardar a maturação e a reduzir a incidência de doenças e distúrbios fisiológicos (Wills et al., 1998).

O "bitter pit", importante distúrbio fisiológico que ocorre em maçãs, geralmente se desenvolve durante a fase de frigoconservação, sendo caracterizado por manchas pequenas de cor escura na casca, acima das áreas de tecido necrosado da polpa. As células mortas da polpa perdem umidade e criam uma pequena depressão na superfície da área atingida. Frutos com teores baixos de $\mathrm{Ca}$ e relações altas de $\mathrm{K} / \mathrm{Ca}$, $\mathrm{K}+\mathrm{Mg} / \mathrm{Ca}$ e $\mathrm{N} / \mathrm{Ca}$ apresentam elevada suscetibilidade ao "bitter pit" (Argenta \& Suzuki, 1994; Ferguson \& Walkins, 1989; Nachtigall \& Freire, 1998).

O movimento de Ca para o fruto ocorre com o suprimento de água, através do xilema, especialmente durante a fase de divisão celular, que ocorre até cerca de 40 dias após a plena floração. Após este período, o suprimento de água para o fruto passa a ser via floema, no qual a mobilidade do Ca é muito baixa (Taiz \& Zeiger, 1998). Este curto período de maior suprimento de $\mathrm{Ca}$ faz com que ocorra diluição do conteúdo deste nutriente com o crescimento dos frutos, o que pode comprometer a qualidade durante o armazenamento.

Os efeitos positivos do Ca na preservação da qualidade póscolheita têm sido atribuídos ao fato de o mesmo estar associado com as substâncias pécticas da lamela média e com as membranas celulares, conferindo rigidez aos tecidos e preservando as características de permeabilidade seletiva do sistema de membranas celulares (Ferguson \& Walkins, 1989; Poovaiah et al., 1988). Além disto, o Ca pode complexar- se com proteínas, como é o caso da calmodulina, a qual apresenta papel regulatório de diversas enzimas (Taiz \& Zeiger, 1998).

A cultivar Catarina foi obtida a partir do cruzamento entre as cultivares Fuji e PWR37T133, sendo recomendada para plantio em regiões com altitude acima de $1.200 \mathrm{~m}$ (Camilo \& Denardi, 2002). A cultivar Catarina é resistente à sarna, tardia, e apresenta frutos com boa conservação em câmaras frigoríficas, porém altamente suscetíveis ao "bitter pit" (Camilo \& Denardi, 2002), podendo manifestar o distúrbio em précolheita.

A análise dos conteúdos de $\mathrm{Ca}, \mathrm{Mg}, \mathrm{K}$ e $\mathrm{N}$ antes da colheita é a forma mais indicada para avaliar o risco de ocorrência de "bitter pit" em maçãs (Ferguson \& Walkins, 1989; Argenta \& Suzuki, 1994; Nachtigall $\&$ Freire, 1998). A parte do fruto a ser analisada, portanto, pode influenciar na predição do risco de ocorrência de "bitter pit". O método atualmente empregado consiste na retirada de uma fatia longitudinal do fruto (menos pedúnculo e semente), contendo tecidos de casca e polpa. $\mathrm{O} \mathrm{Ca}$ apresenta grande variação de teor dentro do próprio fruto. $\mathrm{O}$ teor de $\mathrm{Ca}$, em uma seção radial, é superior na casca e no centro do fruto, e inferior no córtex, sendo que os menores teores estão no córtex exterior do fruto, onde justamente ocorre o "bitter pit" (Terblanche et al., 1979). Existe ainda um gradiente longitudinal, onde o teor de Ca diminui do pedúnculo para o cálice (Ferguson \& Watkins, 1983). Portanto, o método tradicional de amostragem (fatia longitudinal de casca+polpa), envolvendo tecidos com níveis nutricionais bastante diferenciados, pode reduzir a capacidade de predição do risco de "bitter pit". Deste modo, a amostragem de forma individualizada dos tecidos da casca e da polpa, e a análise mineral dos mesmos podem permitir a identificação do tecido e do atributo mineral que melhor discrimina os frutos quanto à severidade de "bitter pit".

Métodos de análise univariada são normalmente empregados visando a identificar diferenças entre grupos de frutos com diferentes graus de severidade ao "bitter pit", considerando individualmente cada

${ }^{1}$ (Trabalho 134/2005). Recebido: 26/07/2005. Aceito para publicação: 06/04/2005.

2 Ph.D., Bolsista do CNPq, Centro de Ciências Agroveterinárias (CAV), Universidade do Estado de Santa Catarina (UDESC), Cx. Postal 281, CEP 88502-970, Lages-SC. Autor para correspondência. E-mail: amarante@cav.udesc.br

${ }^{3}$ Eng. Agr., Aluna do Curso de Mestrado em Produção Vegetal, Bolsista da CAPES. Centro de Ciências Agroveterinárias (CAV), Universidade do Estado de Santa Catarina (UDESC), Cx. Postal 281, CEP 88502-970, Lages-SC. E-mail: a6dvc@cav.udesc.br.

${ }^{4}$ Ph.D., Bolsista do CNPq, Centro de Ciências Agroveterinárias (CAV), Universidade do Estado de Santa Catarina (UDESC), Cx. Postal 281, CEP 88502-970, Lages-SC.E-mail: a2pre@cav.udesc.br. 
atributo mineral. Estes métodos de análise não consideram o efeito conjunto de todos os atributos. A análise estatística multivariada, como é o caso da análise canônica discriminante (ACD), é uma ferramenta que permite a identificação de diferenças existente entre grupos de frutos com diferentes níveis de ocorrência de "bitter pit", considerando todos os atributos nutricionais (Cruz-Castillo et al., 1994). Por tais características, a análise multivariada representa uma ferramenta importante no estudo e identificação do(s) atributo(s) mineral(is) que melhor discrimina(m) níveis de severidade ao "bitter pit". Isto pode permitir ainda a eliminação do tempo gasto com a avaliação de atributos nutricionais que expressam baixa resposta na segregação de frutos quanto ao grau de ocorrência de "bitter pit".

Este trabalho objetivou identificar os nutrientes e/ou relações nutricionais que afetam a severidade de "bitter pit" em maçãs da cultivar Catarina, bem como identificar a porção do fruto (polpa ou casca) a ser amostrada, para que a análise mineral melhor exprima a severidade do distúrbio nesta cultivar. Além de análises de regressões lineares e nãolineares entre atributos nutricionais e severidade de "bitter pit", foi realizada, neste trabalho, a análise canônica discriminante (ACD), visando a identificar o(s) atributo(s) mineral(is) que melhor discrimina(m) frutos com diferentes níveis de severidade ao "bitter pit".

\section{MATERIAL E MÉTODOS}

Maçãs da cultivar Catarina foram colhidas na maturação comercial (com valores de firmeza de polpa, sólidos solúveis totais e acidez titulável de $76,7 \mathrm{~N}, 15,9^{\circ}$ Brix e $0,41 \%$ de ácido málico, respectivamente) em pomar localizado no município de São Joaquim-SC, na safra 20002001, e divididas em quatro lotes de 14 frutos, cada lote com diferentes níveis de severidades ao "bitter pit": 1) nula (nenhuma lesão/fruto); 2) baixa (1-2 lesões/fruto); 3) moderada (3-5 lesões/fruto), e 4) alta (6-18 lesões/fruto). As concentrações de $\mathrm{Ca}, \mathrm{K}, \mathrm{Mg}$ e $\mathrm{N}$ foram determinadas nos tecidos da casca e da polpa de cada um dos frutos.

Três gramas de cada amostra de tecido (da casca e da polpa) foram pesados e transferidos para tubos de digestão, aos quais foram adicionados $2 \mathrm{~mL}$ de $\mathrm{H} 2 \mathrm{SO} 4$ e $3 \mathrm{~mL}$ de $\mathrm{H} 2 \mathrm{O} 2$ (30 volumes). Em seguida, os tubos foram transferidos para bloco digestor ajustado a uma temperatura inicial de $150 \mathrm{oC}$, a qual foi sendo elevada gradativamente de $50 \mathrm{em}$ $50^{\circ} \mathrm{C}$ a cada dez minutos até atingir a temperatura final de $350^{\circ} \mathrm{C}$. A partir desse momento, as amostras foram mantidas no bloco digestor por três horas, sendo que, na metade desse tempo, foram acrescentados $3 \mathrm{~mL}$ de $\mathrm{H} 2 \mathrm{O} 2 \mathrm{em}$ cada tubo. Ao término do processo de digestão, as amostras foram retiradas do bloco digestor e, após resfriarem, tiveram o volume completado para $20 \mathrm{~mL}$ com água destilada. $\mathrm{O} \mathrm{N}$ foi determinado pelo método semimicro-Kjeldahl, como descrito por Tedesco et al. (1995); K, $\mathrm{Ca}$ e $\mathrm{Mg}$ foram determinados por espectrofometria de emissão induzida por plasma. As concentrações dos nutrientes foram expressas em mg $\mathrm{kg}-1$ de massa fresca.

Os dados obtidos foram submetidos à análise estatística, utilizando o programa SAS, versão 6.12 (SAS Institute, Inc.) (1990). Foram realizadas análises de regressão linear e não-linear entre valores médios de atributos nutricionais e de manchas por fruto quantificados nas diferentes categorias de severidade de "bitter pit". Os dados foram também submetidos à análise canônica discriminante (ACD), visando a identificar o(s) atributo(s) mineral(is) que melhor discrimina(m) as diferenças quanto ao grau de suscetibilidades ao "bitter pit" em maçãs 'Catarina'. Os valores médios dos coeficientes canônicos padronizados (CCP) de frutos correspondentes aos diferentes níveis de severidades ao "bitter pit" foram comparados através do teste de Tukey $(\mathrm{P}<0,05)$, conforme descrito por Cruz-Castillo et al. (1994).

\section{RESULTADOS E DISCUSSÃO}

\section{Análises de regressões lineares e não-lineares}

No tecido da casca, houve uma relação linear negativa $(\mathrm{P}<$ $0,05)$ entre os valores médios de Ca na casca e os valores médios de manchas/fruto nas diferentes categorias de severidade de "bitter pit" (Figura 1). É necessário considerar que os valores de erro-padrão da média para as concentrações de Ca na casca, em cada categoria de severidade de "bitter pit", foram elevados, especialmente nos níveis de menor severidade (Figura 1).

A severidade de "bitter pit" aumentou significativamente com o incremento nos valores das relações $(\mathrm{K}+\mathrm{Mg}) / \mathrm{Ca}$ e $(\mathrm{K}+\mathrm{Mg}+\mathrm{N}) / \mathrm{Ca}$ na casca. Houve uma relação linear positiva $(\mathrm{P}<0,05)$ entre os valores médios das relações $\mathrm{Mg} / \mathrm{Ca},(\mathrm{K}+\mathrm{Mg}) / \mathrm{Ca}$ e $(\mathrm{K}+\mathrm{Mg}+\mathrm{N}) / \mathrm{Ca}$ na casca e o número médio de manchas/fruto nas diferentes categorias de severidade de "bitter pit" (Figura 1). A relação $\mathrm{Mg} / \mathrm{Ca}$ na casca apresentou menores valores de erro-padrão da média nas diferentes categorias de severidade de "bitter pit" (Figura 1). Não houve diferenças quanto aos valores de $\mathrm{K} / \mathrm{Ca}$ e N/Ca na casca entre frutos com diferentes níveis de severidade ao "bitter pit" (dados não apresentados).

Houve uma relação exponencial inversa entre os valores médios de Ca na polpa e o número médio de manchas/fruto nas diferentes categorias de severidade de "bitter pit" (Figura 1). Esta relação mostra um aumento substancial na severidade de "bitter pit" em frutos com concentrações de Ca na polpa menores que $32 \mathrm{mg}$ kg-1. Não foi observada associação entre os valores das relações $\mathrm{K} / \mathrm{Ca}, \mathrm{Mg} / \mathrm{Ca},(\mathrm{K}+\mathrm{Mg}) / \mathrm{Ca}$, $\mathrm{N} / \mathrm{Ca}$ e $(\mathrm{K}+\mathrm{Mg}+\mathrm{N}) / \mathrm{Ca}$ na polpa e a severidade de "bitter pit" (dados não apresentados).

\section{Análise multivariada}

$\mathrm{Na}$ análise canônica discriminante (ACD), o número máximo de funções canônicas discriminantes obtido é dado pelo menor valor, calculado em função do número de grupos estudados menos 1 , e do número de atributos avaliados menos 1 (Cruz-Castillo et al., 1994). Como foram estudados 18 atributos nutricionais e apenas quatro níveis de severidade, foi possível a utilização de até três funções canônicas discriminantes. A primeira função canônica discriminante explicou $60 \%$ da variação total, enquanto a segunda e a terceira funções explicaram apenas $21 \%$ e $19 \%$ da variação total, respectivamente. Desta forma, como a primeira função canônica discriminante (FCD1) explicou a maior parte da variação total, apenas esta foi considerada na análise multivariada dos dados obtidos.

O teste estatístico multivariado Wilks Lambda mostrou haver diferenças altamente significativas entre frutos pertencentes aos diferentes níveis de severidade de "bitter pit" para a FCD1 ( $<<0,05)$. AFCD1 apresentou correlação canônica de 0,73 , indicando elevada associação entre os atributos nutricionais estudados e os níveis de severidade de "bitter pit".

O parâmetro adotado para avaliar o efeito de separação gerada pelos atributos nutricionais dentro dos níveis de severidade estudados foi o coeficiente da taxa de discriminação paralela (TDP). O coeficiente da TDP é obtido por meio do produto entre valores dos coeficientes canônicos padronizados (CCP) e os coeficientes de correlação canônica (r). Para o coeficiente da TDP, valores de atributos nutricionais positivos indicam efeito de separação entre os níveis de severidade de "bitter pit", sendo que os atributos com os maiores valores apresentam maior peso na separação entre níveis de severidade. Valores negativos expressam efeito de supressão do atributo na separação entre os níveis de severidade de "bitter pit", ou seja, expressam semelhanças entre os níveis de severidade.

A relação $\mathrm{Mg} / \mathrm{Ca}$ na casca apresentou o maior valor de coeficiente da TDP (Tabela 1), indicando que, dentre todos os atributos nutricionais avaliados nos tecidos da casca e da polpa, este atributo é o que melhor discrimina frutos com diferentes níveis de severidade ao "bitter pit". A relação K/Ca na polpa e a concentração de $\mathrm{Mg}$ na polpa apresentaram menor grau de importância na discriminação entre lotes de frutos com diferentes níveis de severidade ao "bitter pit" (Tabela 1). A análise multivariada mostrou que o valor isolado da concentração de $\mathrm{Ca}$, na casca ou na polpa, não foi relevante na discriminação de lotes de frutos com diferentes níveis de severidade ao "bitter pit". Isto confirma observações realizadas por outros autores, mostrando que relações en- 

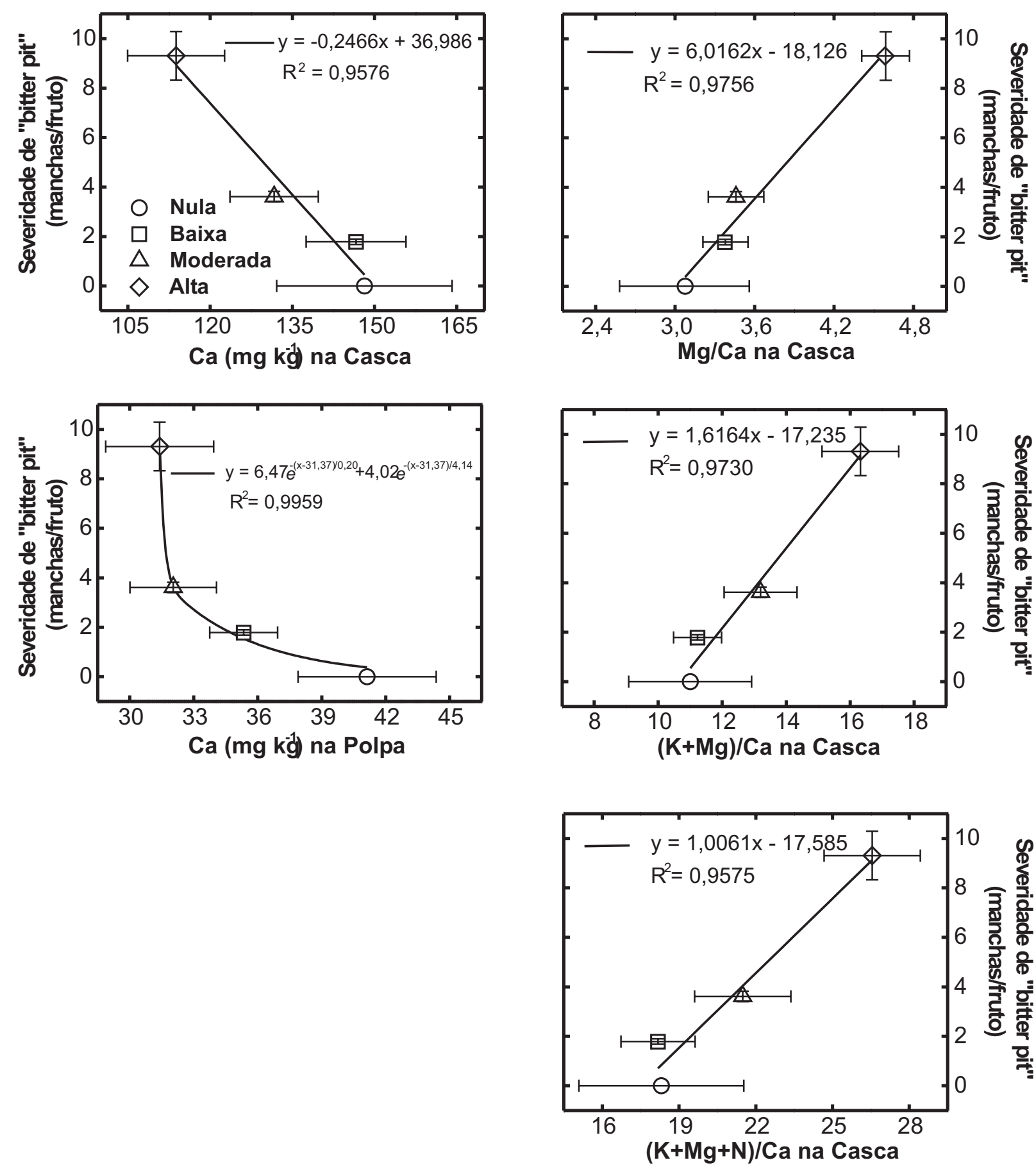

FIGURA 1 - Relações entre os valores médios de atributos nutricionais e o número de manchas por fruto em maçãs 'Catarina' com severidade nula (nenhuma lesão/fruto); baixa (1-2 lesões/fruto); moderada (3-5 lesões/fruto), e alta (6-18 lesões/fruto) de “bitter pit”. Barras horizontais e verticais representam, respectivamente, o erro-padrão da média para os valores de atributos nutricionais e de número de manchas por fruto em cada nível de severidade de "bitter pit”. São Joaquim-SC, 2001.

TABELA 1 - Coeficiente da taxa de discriminação paralela (TDP) para a função canônica discriminante $1\left(\mathrm{FCD}_{1}\right)$, referente às análises dos nutrientes na casca e na polpa. São JoaquimSC, 2001.

\begin{tabular}{ccc}
\hline ATRIBUTOS AVALIADOS & CASCA & POLPA \\
\hline $\mathrm{Ca}$ & $-0,0357$ & $-1,0717$ \\
$\mathrm{Mg}$ & $-0,0202$ & $\mathbf{0 , 5 5 0 8}$ \\
$\mathrm{K}$ & 0,0771 & $-0,0497$ \\
$\mathrm{~N}$ & $-0,0264$ & 0,0535 \\
$\mathrm{~K} / \mathrm{Ca}$ & 0,0259 & $\mathbf{0 , 7 0 5 6}$ \\
$\mathrm{Mg} / \mathrm{Ca}$ & $\mathbf{1 , 7 3 1 2}$ & 0,0265 \\
$\mathrm{~N} / \mathrm{Ca}$ & 0,0000 & 0,6725 \\
$(\mathrm{~K}+\mathrm{Mg}) / \mathrm{Ca}$ & 0,0000 & 0,0000 \\
$(\mathrm{~K}+\mathrm{Mg}+\mathrm{N}) / \mathrm{Ca}$ & $-1,6393$ & 0,0000 \\
\hline
\end{tabular}

tre teores de Ca e de Mg e K são melhores indicadores de suscetibilidade ao "bitter pit" do que teores isolados de Ca (Argenta \& Suzuki, 1994;
Ferguson \& Watkins, 1989; Nachtigall \& Freire, 1998).

A análise multivariada mostrou que a relação $\mathrm{Mg} / \mathrm{Ca}$ na casca é o atributo mineral que melhor discrimina frutos da cultivar 'Catarina' com diferentes níveis de severidade ao "bitter pit", possivelmente por apresentar menor variabilidade entre frutos pertencentes a cada lote e/ou por apresentar maior variabilidade entre lotes de frutos com diferentes severidades ao "bitter pit". Portanto, na cultivar Catarina, a ocorrência de "bitter pit" está fortemente ligada a uma elevada relação $\mathrm{Mg} / \mathrm{Ca}$ no tecido da casca dos frutos.

A representação gráfica entre os coeficientes canônicos padronizados (CCP) das funções canônicas discriminantes 1 e 2 mostra uma nítida separação entre frutos com níveis de severidade nula, baixamoderada e alta (Figura 2). Houve diferença altamente significativa $(\mathrm{P}<$ $0,0001)$ entre os valores médios dos CCP da função canônica discriminante 1 (FCD1) para os diferentes níveis de severidade ao "bitter pit" (Tabela 2). Frutos com alta severidade apresentaram os maiores valores médios de CCP (indicando especialmente frutos com alta relação 
$\mathrm{Mg} / \mathrm{Ca}$ na casca), ao passo que frutos com severidade nula apresentaram os menores valores de CCP (indicando frutos com baixa relação $\mathrm{Mg}$ / Ca na casca). Não houve diferença significativa entre frutos com severidade baixa e moderada (Tabela 2).

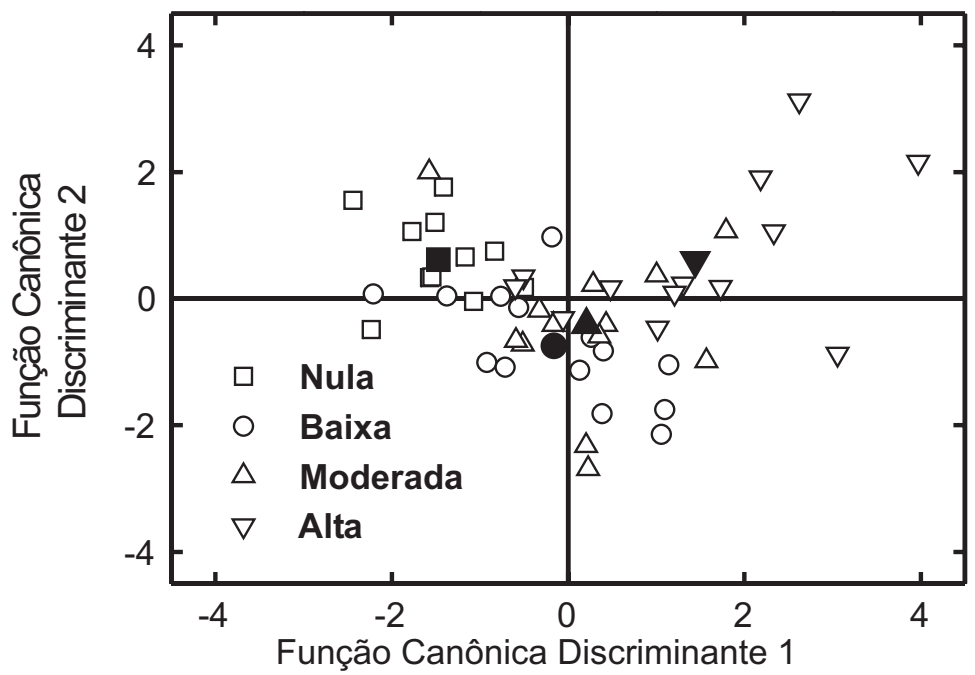

FIGURA 2 - Coeficientes canônicos padronizados (CCP) das funções canônicas discriminantes 1 e 2, em maçãs 'Catarina' com diferentes níveis de severidade ao "bitter pit" (nula: nenhuma lesão/fruto; baixa: 1-2 lesões/fruto; moderada: 3-5 lesões/fruto, e alta: 6-18 lesões/fruto), considerando todos os atributos nutricionais avaliados nos tecidos da polpa e da casca. Símbolo cheio representa o valor médio de CCP para cada nível de severidade. São Joaquim-SC, 2001.

TABELA 2 - Valores médios dos coeficientes canônicos padronizados (CCP) para a função canônica discriminante $1\left(\mathrm{FCD}_{1}\right)$, referente às análises nutricionais de maçãs 'Catarina' com diferentes níveis de severidade ao "bitter pit". São Joaquim-SC, 2001.

\begin{tabular}{lc}
\hline Severidade $^{\mid}$ & CCP \\
\hline Nula & $-1,4719 \mathrm{c}^{2}$ \\
Baixa & $-0,1615 \mathrm{~b}$ \\
Moderada & $0,2057 \mathrm{~b}$ \\
Alta & $1,4402 \mathrm{a}$ \\
\hline
\end{tabular}

${ }^{1}$ Severidade nula: nenhuma lesão/fruto; baixa: 1-2 lesões/fruto; moderada: 3-5 lesões/fruto, e alta: 6-18 lesões/fruto. ${ }^{2}$ Médias nas colunas seguidas da mesma letra não diferem entre si, pelo teste de Tukey $(P<0,05)$.

\section{CONCLUSÕES}

Os resultados deste trabalho permitem concluir que o aumento na severidade de "bitter pit" na cultivar Catarina é o resultado de baixas concentrações de Ca nos tecidos da casca e da polpa, bem como dos elevados valores das relações $\mathrm{Mg} / \mathrm{Ca},(\mathrm{K}+\mathrm{Mg}) / \mathrm{Ca}$ e $(\mathrm{K}+\mathrm{Mg}+\mathrm{N}) / \mathrm{Ca}$ na casca. No entanto, a análise multivariada (ACD) mostra que o aumento na severidade de "bitter pit" nesta cultivar é melhor explicado pela incremento na relação $\mathrm{Mg} / \mathrm{Ca}$ no tecido da casca.

\section{AGRADECIMENTOS}

Os autores agradecem ao Conselho Nacional de Desenvolvimento Científico e Tecnológico (CNPq) e à Coordenação de Aperfeiçoamento de Pessoal de Nível Superior (CAPES) o apoio financeiro a este projeto.

\section{REFERÊNCIAS}

ARGENTA, L.C.; SUZUKI, A. Relação entre teores minerais e freqüência de bitter pit em maçã cv. Gala no Brasil. Revista Brasileira de Fruticultura, Cruz das Almas, v. 16, n. 1, p. 267-277, 1994.

CAMILO, A.P.; DENARDI, F. Cultivares: descrição e comportamento no sul do Brasil. In: EPAGRI. A cultura da macieira. Florianópolis, 2002. p. 113-168.

CRUZ-CASTILLO, J.G.; GANESHANANDAM, S.; MACKAY, B.R.; LAWES, G.S.; LAWOKO, C.R.O.; WOOLLEY, D.J. Appications of canonical discriminant analysis in horticultural research. HortScience, Alexandria, v. 29, n. 10, p. 1115-1119, 1994.

FERGUSON, I.B.; WATKINS, C.B. Cation distribution and balance in apple fruit in relation to calcium treatments for bitter pit. Scientia Horticulturae, Amsterdam, v.19, n. 3/4, p.301-310, 1983.

FERGUSON, I. B; WATKINS, C. B. Bitter-pit in apple fruit. Horticultural Reviews, New York, v. 11, p. 289-355, 1989.

NACHTIGALL, G.R.; FREIRE, C.J.S. Previsão da incidência de "bitter pit" em maçãs através dos teores de cálcio em folhas e frutos. Revista Brasileira de Fruticultura, Cruz das Almas, v. 20, n. 2, p. 158-166, 1998.

POOVAIAH, B.W.; GLENN, G.M.; REDDY, A.S.N. Calcium and fruit softening: physiology and biochemistry. Horticultural Reviews, New York, v. 10, p. 107-152, 1988.

SAS. SAS institute INC. Cary, NC, 1990.

TAIZ, L.; ZEIGER, E. Plant physiology. 2nd ed. Sunderland: Sinauer Associates, 1998. 793p.

TEDESCO, M. J.; GIANELLO, C.; BISSANI, C. A.; BOHNEN, H.; VOLKWEISS, S. J. Análise do solo, planta e outros materiais. 2. ed. Porto Alegre: Departamento de Solos, UFRGS, 1995. 174 p. (Boletim Técnico de Solos, 5).

TERBLANCHE, J.H.; GÜRGEN, K.H.; PEINAR,W.J. Concentration gradients of $\mathrm{K}, \mathrm{Ca}$ and $\mathrm{Mg}$ in Golden Delicious apples with reference to bitter pit. Deciduous Fruit Grower, Belville, v. 29, n. 2, p. 76-79, 1979.

WILLS, R. H.; McGLASSON, W. B.; GRAHAM, D.; JOYCE, D. Postharvest, an introduction to the physiology and handling of fruit, vegetables and ornamentals. 4th ed. New York: CAB International, 1998.262p. 